\title{
Mindfulness Meditation Influences Implicit but Not Explicit Coding of Temporal Simultaneity
}

\author{
Mark A. Elliott ${ }^{1} \cdot$ Monika Zalewska $^{1} \cdot$ Marc Wittmann $^{2}$
}

Received: 10 April 2021 / Accepted: 27 September 2021 / Published online: 11 October 2021

(c) The Author(s) 2021, corrected publication 2022

\begin{abstract}
In the meditative state time appears to slow down and in the present moment it expands. However, to date, there is no investigation of the effect of meditative state on the structure of the "psychological moment"; this is the measurable, minimal duration of the moment "now." In this study, we examined the effect on the psychological moment of a mindfulness intervention against an intervention in which participants listened to classical music. The psychological moment was measured using a simultaneity-detection paradigm from which the threshold between reports that two targets changed luminance simultaneously or with an asynchrony is normally taken as the duration of the moment. In line with previous research, this paradigm allowed for examination of the effects of the subthreshold synchronized, or asynchronized target onsets, which occurred prior to the luminance change of the targets. While there was no overall difference in the psychological moment pre- and post-, and as a function of the type of intervention, a bias against reporting simultaneity following presentation of a subthreshold asynchrony, which lowered thresholds and so shortened the psychological moment, was reduced after the mindfulness intervention. From this we conclude that even brief mindfulness meditation can encourage a more focalized attentional response, which can in turn be used to normalize psychological time.
\end{abstract}

Keywords Mindfulness $\cdot$ Meditation $\cdot$ Low-level vision $\cdot$ Implicit visual processing

\section{Introduction}

Mindfulness meditation stems from the Buddhist Theravada tradition and is considered an altered state of consciousness (Vaitl, et al., 2005), which focuses on cultivating a non-judgmental awareness of the present moment through purposefully paying attention to both external and internal sensory stimuli (Kabat-Zinn, 1994, 2003). Several studies have investigated the possible effects of mindfulness meditation on the perception of time (Berkovich-Ohana et al., 2013; Wittmann \& Schmidt, 2014; Wittmann, et al., 2015). Initially, meditators experience a subjective slowing of the passage of time and the expansion of the present moment (referred to here as the "psychological present"). This is achieved through a transiently increased attentional focus

Mark A. Elliott

mark.elliott@nuigalway.ie

1 School of Psychology, National University of Ireland, Galway University Road, Galway, Republic of Ireland

2 Institute for Frontier Areas in Psychology, Freiburg i Br, Germany on body states (Matko et al., 2021), which leads meditators to be more strongly aware of their bodily self, which in turn slows down, subjectively, the passage of time (Wittmann, 2015). An increased awareness of oneself coincides with an increased awareness of time. It is suggested that this altered sensitivity to the passage of time is achieved through increased momentary awareness of sensory experiences, better emotion regulation, and heightened attentional resources (Hölzel et al., 2011). Being in a peak of the meditative state however leads experienced meditators to the transient disappearance of the self and time (Alexander et al., 1991; Berkovich-Ohana \& Wittmann, 2017; Droit-Volet \& Dambrun, 2019). Depending partly on the type of meditation, and also for novice meditators, time in retrospect feels comparatively shorter when attention is continuously focused on an object of meditation, rather than when monitoring self-related perceptions and emotions (Sedlmeier et al., 2020).

Regarding mindfulness meditation, two studies have shown that the level of mindfulness experience was positively correlated with an overestimation of duration (Kramer et al., 2013), with less time pressure, a sense that the psychological present had expanded, and the experience of 
time passing more slowly (Wittmann et al., 2015). In a third study, Sauer et al., (2012) found that practicing meditators were able to hold a switching percept of the Necker cube, a visually ambiguous figure, for longer than non-meditators. This shows that meditators, due to their deeper levels of meditative state, were able to focus their attention for longer, leading to the experience of an expanded psychological present. In another study, time perception in the meditation session itself was measured, during which all subjects listen to relaxing music and subsequently practice Shavasana yogic mindfulness (Thönes \& Wittmann, 2016). Thönes and Wittmann found that the subjective passage of time was significantly faster during yogic meditation than in a control condition, and it was positively correlated with the level of experienced relaxation.

Not only does long-term practice of mindfulness meditation leads to altered time perception, in a recent study on dispositional mindfulness, Wittmann et al. (2014) showed that individuals who reported being more mindful in daily life were less impulsive and more accurate in the estimation of physical time in the milliseconds-to-seconds range. The claim that mindfulness influences time perception is not conclusive: Droit-Volet and Heros (2017) found no differences in judgments of intervals between events in the range $800 \mathrm{~ms}(\mathrm{~ms})$ to $2 \mathrm{~s}(\mathrm{sec})$, between novice and experienced meditators before and after a period of meditation. A similar pre-and post-meditation design also found no effect of quickly induced meditative state on performance in a temporal production task using stimuli presented over 4- to 32-s durations (Berkovich-Ohana et al., 2011).

The temporal organization of sensory and psychological experiences lies at the core of self-identity (Husserl, 1928), and on this basis, one might conclude that an appropriate coding of temporal events is necessary for healthy establishment of the self and understanding of the world around. In altered states of consciousness (ASCs), such as during the meditative state, or in neurological disorders, the perception of time and sense of self is changed (Berkovich-Ohana \& Wittmann, 2017; Vaitl et al., 2005) and in this context, patients with schizophrenia experience an "abnormal sense of self" where they feel disconnected from the reality and themselves. It is argued that this is caused by the distortions of the temporal structure of consciousness (Giersch et al., 2013, 2015).

Elsewhere it is argued that studies of time perception may not be a valid means to examine variations in the temporal structure of processes that contribute to a sense of temporality, or time passage (Elliott, 2019). With this issue in mind, Elliott et al. (2007) developed a paradigm, based upon and replicating the earlier work of Brecher (1932), which showed remarkable consistency in measures of the "minimal" psychological moment in the range 50-60 ms. The psychological moment according to Brecher, and Elliott and colleagues describes the minimum interval of time within which events appear in temporal coincidence. As such, the psychological moment is equated with "nowness" or the psychological present, with the duration of the psychological moment being equivalent to the minimum duration of the moment now. A body of literature, reviewed by Elliott and Giersch (2016), has examined both variations in the duration of the psychological present, as a function of psychopathology (i.e., Giersch, et al., 2009; Schmidt et al., 2011), and the psychophysical structures responsible for organizing events into a single moment, which appear to be serial and not parallel in their microstructure as one might expect. However, as regards the duration of the psychological present in healthy volunteers, there are two important aspects to the paradigm developed by Elliott et al. (2007): the first of these is that the psychophysical measure of the psychological present is derived from judgments of the simultaneity or asynchrony of the change in luminance of two stimulus bars presented on a computer screen. The logic in this case is that the present moment, in which all events are co-temporal, may be measured for so long as these changes in luminance appear to the observer to be co-temporal. In healthy participants, co-temporality usually extends to event asynchronies in the range 55-60 ms, after which these events are more often reported as asynchronous rather than simultaneous. In this respect, there is little scope for bias or the overinterpretation of memory or attentional effects in terms of time perception (see Grondin, 2001 for a review as well as Elliott et al., 2006, for a development on these ideas). This measure of the duration of the psychological present is thereby indirect (calculated over a range of asynchronies sampled using a repeated measures, method of constant stimuli), and any variation as a function of mindfulness meditation may address a possible issue of validity that is raised by some studies, namely that the effects of meditation on explicit time estimation are particular to experienced mediators (see e.g. Carter et al., 2005; Droit-Volet et al., 2015).

The second aspect of the paradigm developed by Elliott et al. (2007) concerns the below-threshold presentation of the stimulus bars that subsequently change luminance for the purpose of the asynchronous/simultaneous report. The bars are initially presented within the context of the rapid presentation and switching off of flanking bars, which has the effect of rendering both the presentation of the bars and whether or not their presentation was asynchronous or synchronous, as not reliably reportable by observers. In spite of being non-reportable, some studies (Giersch et al., 2009; Schmidt et al., 2011, Elliott \& Shanagher, 2010) have found that asynchronous presentation significantly reduces the simultaneity threshold relative to synchronous presentation, while Elliott et al. (2007) reported that bar-presentation asynchrony was significantly biased against reporting the subsequent luminance change as simultaneous. This occurred only for very short asynchronies 
between luminance changes and was matched by a corresponding bias to report simultaneity at longer asynchronies, while the threshold remained uninfluenced. In other words, the synchrony or asynchrony of bar presentation, although not reportable, leads to a change in the slope of the psychometric function associated with reports of bar luminance change simultaneity or asynchrony. Elliott et al. speculated that very early visual mechanisms concerned with bar-bar binding might be influenced by this aspect of the paradigm. In the present context, if there is no overall variation in simultaneity thresholds as a function of mindfulness meditation, the presence of the bias described here alongside both changes in threshold and changes in the slope of the psychometric function would be examined.

The overall goal of our study is to examine the effect of mindfulness meditation on the psychological moment using the paradigm developed by Elliott et al. (2007), while a secondary aim is to investigate the relationship between dispositional mindfulness and impulsiveness, to compare possible correlations with findings of other studies (Wittmann \& Paulus, 2008). Our overall goal could be subdivided into two questions: first, we aimed to establish whether or not the duration of the psychological moment is particularly influenced by mindfulness meditation. If this were found to be the case, the simultaneity thresholds derived from analysis of data obtained from post-mindfulness meditation intervention should differ from those taken pre-intervention as well as those taken from a control group who received a non-mindfulness meditation intervention. The second aim concerns previous reports of a bias to report asynchrony when the target bars changed luminance following their asynchronous presentation within a premask of flanking bar presentations: although this did not influence simultaneity thresholds, Elliott et al. (2007) found it altered the slope of the psychometric function, indicating a difference in the structure of processing as a result of asynchronous, relative to synchronous bar presentations. Because mindfulness is concerned with the perception of event structures, and the conditioning of attentional mechanisms concerned with perception, we considered whether and how judgments of the simultaneity of bar-bar events are influenced by the "mindfulness conditioning" of early perceptual mechanisms. Guided meditation can have short-term and even long-term effects on individuals who are only beginners of meditation (Mrazek et al., 2012) or have practically no experience at all (Kramer et al., 2013); consequently we aimed to test participants with limited or no experience of mindfulness meditation.

\section{Method}

\section{Participants}

A total of 36 participants, 16 males and 20 females (mean age $M=21.83, S D=5.28$ ), were randomly assigned to the experimental mindfulness meditation group $(n=18)$ or the control classical music group $(n=18)$. All students were recruited at NUI Galway through SONA system. They were over 18 years of age, had a normal or corrected-to-normal vision, and had no previous diagnosis of neurological or psychiatric problems. At the recruitment stage, all participants were asked to confirm that they had less than $30 \mathrm{~h}$ experience of mindfulness meditation. Those who did not meet this criterion were excluded from the participation. Participation was voluntary and students received course credit in exchange for their participation.

\section{Apparatus and Materials}

\section{Questionnaires}

Participants completed basic demographic information (age, sex) along with the two trait measures: dispositional mindfulness and impulsivity (Table 1). Dispositional mindfulness was measured with the Freiburg Mindfulness Inventory (FMI) (Walach et al., 2006) which is a 14-item self-reported questionnaire that includes items rated on a 4-point Likert scale, ranging from 1 (rarely) to 4 (almost always). Higher scores reflect more mindfulness. The questionnaire measures two dimensions of mindfulness: "presence" as an ability to attend to present moment and "acceptance" as a non-judgmental attitude. The inventory was chosen as a psychologically valid instrument (Cronbach's alpha $=0.86$ ) measuring mindfulness trait even in the participants without

Table 1 Mean group differences between the experimental and control group in self-rating scales

\begin{tabular}{|c|c|c|c|c|}
\hline \multirow[t]{2}{*}{ Variable } & \multicolumn{4}{|l|}{ Means (SD) } \\
\hline & Experimental group & Control group & $T(\mathrm{df}=24)$ & $p$ \\
\hline \multicolumn{5}{|c|}{ Freiburg Mindfulness Inventory (FMI) } \\
\hline Presence & $14.00(4.11)$ & $16.14(2.71)$ & 1.59 & .13 \\
\hline Acceptance & $18.83(5.89)$ & $19.93(3.25)$ & 60 & .56 \\
\hline Total FMI & $32.83(9.60)$ & $36.07(5.58)$ & 1.07 & .29 \\
\hline \multicolumn{5}{|c|}{ Barratt Impulsiveness Scale (BIS) } \\
\hline Non-planning & $25.92(5.35)$ & $24.86(4.40)$ & -.55 & .59 \\
\hline Motor & $23.00(4.41)$ & $22.36(2.98)$ & -.44 & .66 \\
\hline Cognitive & $19.25(2.30)$ & $16.43(4.86)$ & -1.74 & .09 \\
\hline Total BIS & $68.17(11.50)$ & $63.64(10.21)$ & -1.06 & .30 \\
\hline
\end{tabular}


previous meditation experience (Sauer et al., 2013; Walach et al., 2006). To measure impulsiveness, we used the Barratt Impulsiveness Scale (BIS-11) (Barratt et al., 1999). The BIS-11 consists of 30 4-point items ranging from 1 (rarely) to 4 (almost always), which are grouped into three subscales: non-planning impulsivity, motor impulsivity, and attention/cognition impulsivity. Higher scores indicate more impulsivity.

\section{Audio Recordings}

During the intervention, participants in the experimental group listened to 2 guided meditation exercises: a body-scan $(10 \min 40 \mathrm{~s})$ and a breathing meditation $(11 \mathrm{~min} 58 \mathrm{~s})$ in this order, while those in the control group listened to the combination of 4 pieces of classical music (total duration 21 min 55 s). During the body-scan meditation, participants were asked to focus attention on each part of the body in order to become aware of the sensations that arise there. In the breathing meditation, attention was focused on natural breathing moment by moment, without judgment of any emergent feelings, thoughts, or sensations (Droit-Volet et al., 2015). We chose this combination of mindfulness exercises as they complement each other and allow for a deeper induction of the meditative state. For the control intervention, we used a special selection of the classical music, with stimulating motives: Canon and Piccola Serenata Notturna, and calming pieces: Piano Concerto (Andante part) and Air. This compilation of stimulating as well as sedative music segments has a more relaxing effect than sedative music alone (Scheufele, 2000), and similarly to the mindfulness meditation, it induces an altered state of consciousness, yet without the component of awareness of the present moment (Schäfer et al., 2013). All audio recordings were presented using AKG Acoustics K 530 closed-back headphones.

\section{Equipment and Stimuli}

The experiment was run on Pentium 4 computer equipped with a Cambridge Research System (Rochester, Kent, UK) visual stimulus generator (ViSaGe). The experimental procedure and stimuli were programmed in the $\mathrm{C}$ programming language using the VSG software library. Stimuli were presented on a Mitsubishi Diamond Pro 2070SB visual display monitor with the vertical refresh rate set to $120 \mathrm{~Hz}$. The monitor was calibrated using a Cambridge Research Systems OptiCAL photometer. Participants were seated in the steady distance of $55 \mathrm{~cm}$ from the monitor, held by means of a chinrest. Stimulus presentation occurred in a soundproof laboratory, in the environment of low-intensity ambient light $\left(0.1 \mathrm{~cd} / \mathrm{m}^{2}\right)$ to reduce the impact of onscreen persistence. The target stimuli were two gray vertical bars which changed luminance on a black background, surrounded by six flankers (see Fig. 1).

\section{Procedure}

Each participant was individually tested in the laboratory room. All participants were asked to choose their time slot for participation in the study such that this time slot would not interfere with periods of high mental activity and/or periods of potential stress (e.g., academic tests/exams/course assessments), in a period up to $2 \mathrm{~h}$ prior to participation. The high level of stress due to this type of mental activity could affect the results of the experiment, hence this request. All participants attended their chosen session between 9 am and $5 \mathrm{pm}$ daily. On the arrival, each participant was randomly
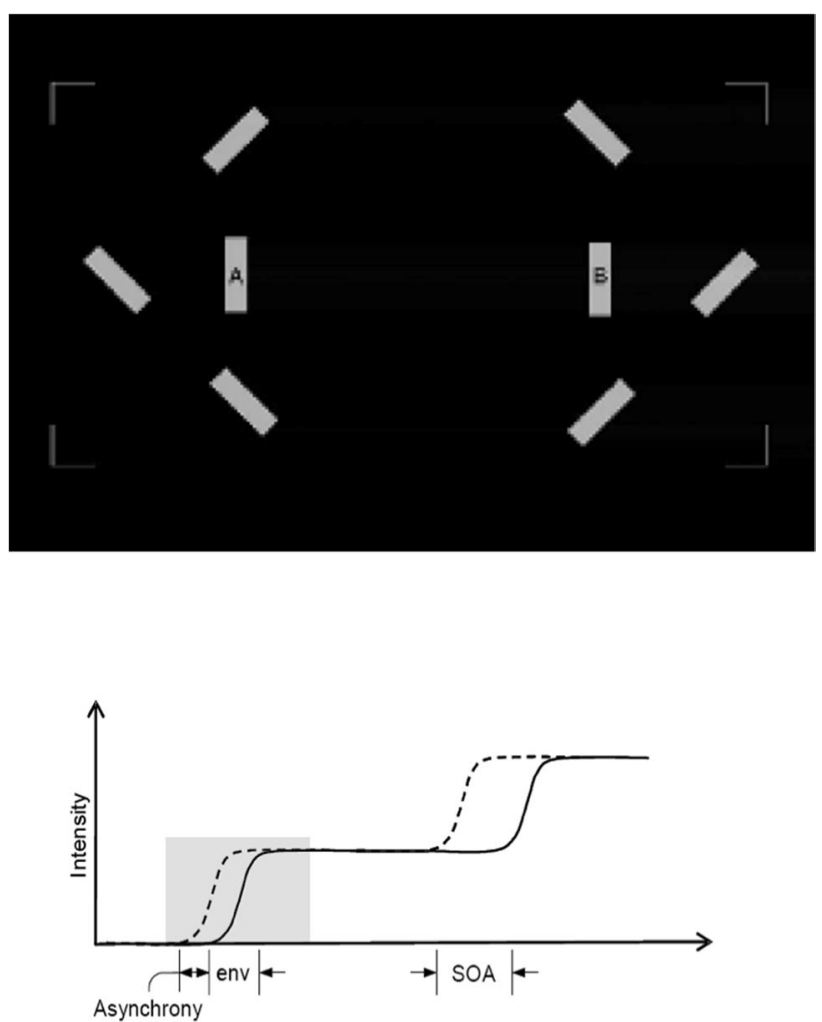

Fig. 1 Upper panel: schematic presentation of the premask condition displayed to participants. Two central bars, indicated as A and B on the picture (letters were not presented during the task), represent the target bars which are surrounded by six pseudo-randomly positioned flankers rotated at $45^{\circ}$ to the left or right of the vertical meridian). Lower panel: a schematic indicating the sequence of target luminance changes in the experiment. The dashed and solid lines represent the luminance of the two target bars. The gray region represents the interval of time during which the dynamic presentation of flankers masked the first change in luminance. Following disappearance of the flankers, the target bars remained on screen with same luminance for $150 \mathrm{~ms}$ and then increased luminance a second time. Participants were asked to report whether or they perceive the second increases as simultaneous or asynchronous 
allocated to either experimental $(n=18)$ or control group $(n=18)$ and this randomization controlled for the potential effect of circadian rhythm on the results (i.e., each participant had equal chance to attend their session at different time during the day and to be assigned to different group). Participants were asked to complete an online form on the computer provided in the laboratory which included basic demographics and the two questionnaires (FMI and BIS-11).

Participants subsequently completed a 4-stage experimental procedure: first, two staircase procedures were carried out to parametrize the range over which the subthreshold onset of target bars could be presented asynchronously, without this change being reportable, and without this change being confusable with the synchronous presentation of the bars (details below). In the second stage of the study, participants undertook the experiment. This used a method of constant stimuli in which a series of trials were presented, and on each trial, they judged the simultaneity or otherwise of the final illumination of two stimulus bars (full details are given below). This was followed by an intervention, in which they underwent audio-guided mindfulness meditation (experimental group) or listened to classical music (control group). Interventions were performed seated in the experimental cabin with the cabin lights on, and participants were asked to follow the guide's instructions as best as they can (mindfulness group), or to relax and listen to the music (control group). Finally, the participants performed the simultaneity judgment task a second time.

\section{Experimental Procedure}

The experiment, including both the staircase procedure and the main experiment, required participants to judge a simultaneity or an asynchrony of two visual stimuli by pressing the "F" on the keyboard if they judged that two visual stimuli occurred simultaneously, and " $\mathrm{J}$ " if they judged that visual stimuli occurred with an asynchrony. Participants were asked to avoid eye movements or blinks during the experimental trials and to judge their response as accurately as possible.

The staircase procedure was run to establish two independent simultaneity thresholds: a "lower" and an "upper." These thresholds were subsequently used to decide the range within which the two target bars were presented asynchronously within a presentation of flanker bars (in a second condition, these bars were presented synchronously). In the case of the higher simultaneity threshold, the target bars were presented in the context of rapidly presented sequence of flanking bars (illustrated in Fig. 1). These flankers masked onset of the target bars and so the derived threshold is equivalent to the maximum interstimulus interval (IS) between bar onsets at which the synchrony or asynchrony of the onsets was not reliably reportable. In the case of the lower simultaneity threshold, the bars were presented without flanking bars, and under these conditions, the derived threshold is the minimum ISI where bar presentations can be reliably judged to be asynchronous. It is this minimum ISI that is not confusable with the physically simultaneous onset of the two bars. Both thresholds were determined using a stochastic approximation procedure developed by Treutwein (1995), in which two bars are first presented with a stimulusonset asynchrony (SOA) either above threshold (at $80 \mathrm{~ms}$ ) and then gradually reduced on each trial until the response "simultaneous" is obtained, or below threshold (at $0 \mathrm{~ms}$ ) and then gradually increased on each trial until the response "simultaneous" is obtained. The lower and upper thresholds were determined separately for each participant.

In the main experiment, bars were presented within a premask comprising a pseudo-randomized sequence of six bars which were rapidly presented and then switched off at locations flanking the two target bars (see Fig. 1). The bars were presented within as the third or fourth event in this sequence either synchronously, or they were presented with an asynchrony within the range of ISIs circumscribed by the lower and upper thresholds (derived from the procedure described above). The target bars remained onscreen, and after a delay of $150 \mathrm{~ms}$ following presentation the final flanker, they changed luminance either simultaneously or at an ISI in the range 10-110 ms. The bars then remained on screen for $2 \mathrm{~s}$ until a response had been made, and the participants were asked to report if the bars had changed luminance simultaneously or with an asynchrony. Bars changed luminance at one of 12 ISIs over the range of $0,10,20$, $30,40,50,60,70,80,90,100$, and $110 \mathrm{~ms}$. Each ISI was presented 40 times with presentation order randomized on an experiment-by-experiment basis. The main experiment consisted of 12 blocks compromising 48 trials per block in which all experimental conditions and trials were pseudorandomly ordered for each participant to control for predictability and habituation. Additionally, the first bar appeared on each side (to the left and to the right) in an equal number of trials. There was an interval of $1 \mathrm{~s}$ between trials and $12 \mathrm{~s}$ between blocks.

\section{Design}

A $2 \times 2 \times 2$ three-way mixed design was employed in the study. The two within-subjects' factors were as follows: time with two levels (pre- and post-intervention) and premask with two levels (synchronous and asynchronous). Premask refers to the appearance of the target bars within the masking sequence which was either synchronized (i.e., both appeared at the same time), or presentation was asynchronous, with the delay between presentation set at a value above the lower simultaneity threshold determined using the staircase procedures (i.e., was not identical to synchronous presentation). The delay 
between presentation was also set below the higher simultaneity threshold, so presentation could not be reliably reported by the participant. The between-subjects factor was group with two levels (experimental and control). The dependent variable was the calculated simultaneity threshold.

\section{Statistical Analysis}

Pearson's correlations were conducted to examine the relationship between the dispositional mindfulness and impulsivity as the total score and on each subscale: the FMI (acceptance, presence) and the BIS (non-planning, motor, cognitive).

Psychometric functions and simultaneity thresholds were calculated individually using a least squares fitting procedure adapted by MATLAB. Initial analysis of the data indicated a high guess rate; thereby individual probability-based corrections were employed to eliminate the problem of a bias towards asynchronous responses. As used by Elliott et al. (2007), the correction was as follows:

$P_{a d j}(\mathrm{x})=\frac{P(x)}{P(0)}$

where $P(0)$ represents the percentage of "synchronous responses" when premasks were presented simultaneously. Simultaneity thresholds were calculated individually at the ISIs corresponding to a rate of $50 \%$ simultaneous responses for synchronous and asynchronous premask separately.

Thresholds were analyzed using a $2 \times 2 \times 2$ ANOVA with the within-subjects factors premask (synchronous vs. asynchronous) and time (pre- vs. post-intervention) and the between-subjects factor group (mindfulness vs. controls). Effects were subject to Bonferroni corrections when appropriate and an alpha of 0.025 was adopted. An ANOVA was conducted on the slopes of the psychometric functions with the factors premask and time (within-subjects) and group (between-subjects) conditions.

\section{Results}

\section{Analysis of Mindfulness and Impulsivity}

The results of the correlational analysis showed that there were no significant correlations between the dispositional mindfulness and impulsivity and on each subscale of the FMI and the BIS (all $p>0.05$ ).

\section{Group Differences in Simultaneity Thresholds}

The data were normally distributed: Kolmogorov-Smirnov and Shapiro-Wilk tests were all non-significant (all $p>0.05$ ), while Box's test of equality of covariance and Levene's test of equality of variance were also non-significant

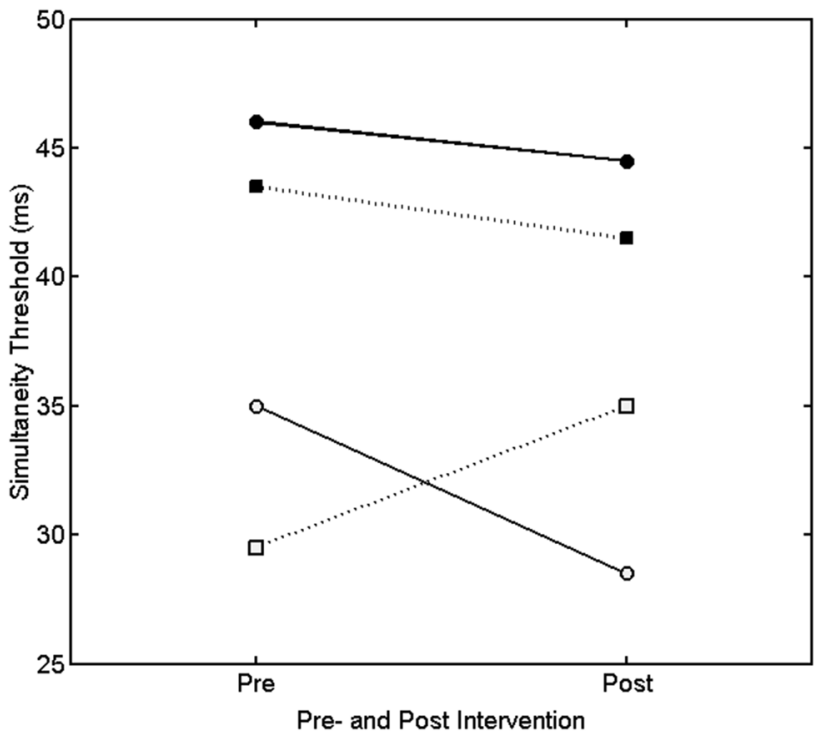

Fig. 2 The interaction of reports differs between Classical Music (continuous line) and Meditation (dashed line) interventions pre- and post-intervention in synchronous-premask (filled symbols) and asynchronous-premask (unfilled symbols) conditions. Although of numerical magnitude, the difference between groups was non-significant. While the difference between premask conditions was significant, the three-way interaction revealed no significant difference between asynchronous and synchronous premasks for the post-mindfulness condition only

for all conditions (all $p>0.05$ ) ensuring homogeneity. Visual analysis of the individual psychometric functions revealed a few unusual response patterns suggesting the data of 10 participants (6 experimental and 4 controls) were likely based upon criteria other than those presented in the experiment (e.g., see-saw responses over ISI or responses that did not exceed 50\% responses "simultaneous" even for the $0 \mathrm{~ms}$ ISI condition, and for which no psychometric function could be calculated). These data were excluded from further analysis. The remaining data from 12 experimental and 14 control participants were considered sufficient given the size of data sets previously reported for this paradigm.

In the ANOVA there was a main effect for premask ( $F$ $\left.(1,24)=69.35, p><0.001, \eta_{\mathrm{p}}^{2}=0.74\right)$ with overall higher simultaneity thresholds in the synchronous $(M=43.76$, $S D=2.40$ ) compared against the asynchronous condition $(M=31.99, S D=2.52)$, with thresholds in both conditions lower than those previously reported (see, e.g., Elliott et al., 2007; Giersch et al., 2009; Schmidt et al., 2011). Additionally, there was a significant three-way, premask by time by group interaction $\left(F_{(1,24)}=7.22, p=0.013, \eta_{\mathrm{p}}^{2}=0.23\right)$ shown in Fig. 2. There were no other significant effects or two-way interactions (all $p>0.1$ ).

Simple main-effects analyses were used to examine the significant three-way interaction, revealing that differences in thresholds following synchronous relative to 
Table 2 Simple-main effects analysis of the differential effect of premask on thresholds, by group and time

\begin{tabular}{llllll}
\hline Group & Time & $\begin{array}{l}\text { D (sync-async) } \\
\text { and SEM (in ms) }\end{array}$ & $F$ & $p$ & Power* \\
\hline Classical music & Pre- & $11(2.4)$ & 21.30 & .000 & .993 \\
& Post- & $16(2.6)$ & 38.63 & .000 & 1.000 \\
\multirow{2}{*}{ Meditation } & Pre- & $14(2.6)$ & 29.22 & .000 & .999 \\
& Post- & $6.5(2.8)$ & 5.72 & .025 & .631 \\
\hline
\end{tabular}

${ }^{*}$ Computed using alpha $=.025$

Table 3 Descriptive statistics of mean thresholds (and their standard deviations) by condition

\begin{tabular}{llll}
\hline & Group & Mean & Std. deviation \\
\hline Pre-Synch & Classical Music & 46 & 13 \\
& Meditation & 44 & 11 \\
Post-Synch & Classical Music & 44 & 12 \\
& Meditation & 41 & 15 \\
Pre-Asynch & Classical Music & 35 & 12 \\
& Meditation & 30 & 14 \\
Post-Asynch & Classical Music & 29 & 16 \\
& Meditation & 35 & 14 \\
\hline
\end{tabular}

asynchronous premasks, post-mindfulness intervention were borderline significant $[F(5.72), p=0.025$, mean difference synchronous minus asynchronous was $6.5 \mathrm{~ms}$ ), while differences were more substantial and substantially more significant for all other time conditions (see Table 2, mean thresholds are given in Table 3). No other contrasting pattern of effects was revealed within the interaction structure.

As shown in Fig. 3, the patterns of synchronous and asynchronous responses after meditation were different with a bias against reporting "simultaneous" substantially reduced in the post-mindfulness intervention condition. An ANOVA on the slopes of the psychometric functions with the factors premask and time (within-subjects) and group (betweensubjects) conditions found slopes between synchronous and asynchronous premask conditions were different $[F$ $\left.(1,96)=36.24, p\rangle<0.001, \eta_{p}^{2}=1\right]$, but failed to resolve the interaction. This suggests that the structure of the perceptual process remains the same irrespective to the type of intervention, although the criteria employed to make the "simultaneous" or "asynchronous" judgment are different following the mindfulness intervention.

A final analysis assessed pre-existing group differences in their level of dispositional mindfulness and impulsivity. No significant differences were found between the experimental and control group on the dispositional mindfulness $(t(24)=1.07, p=0.29)$ or on impulsivity $(t(24)=-1.06$, $p=0.30$ ) (see Table 1). This suggests the different susceptibility to the premask described above it due to state rather than trait mindfulness and therefore a consequence of the mindfulness intervention.

\section{Discussion}

This study investigated for possible variation in the duration of the minimal psychological moment after a brief mindfulness meditation session, with moment duration indexed by the psychophysical threshold between reports that two target bars brightened at the same time (simultaneously), or with a delay. In the event that there were no differences between thresholds in the mindfulness and non-mindfulness control condition, differences in the number of "simultaneous" responses were to be investigated for bias against reporting "simultaneous" based upon the synchrony or asynchrony of masked target bar onset, an effect previously reported by Elliott et al. (2007).

We considered previous research which has found an association between self-attributed mindfulness, impulsivity, and the subjective evaluation of time; i.e., individuals who reported being more mindful in daily life were less impulsive and more accurate in time estimation for intervals of between milliseconds-to-seconds duration in both visual and auditory tasks (Wittman et al. 2014). In addition, other studies have shown a correlation between impulsivity and mindfulness (Weiner et al., 2016; Wittmann \& Paulus, 2008). Impulsiveness is also negatively correlated with time estimation; i.e., less impulsive participants were more accurate in their duration discrimination ability (Wittmann et al., 2014). However, we did not find any correlations between variations in either dispositional mindfulness or impulsivity with variation in thresholds in the present study.

The main findings were that simultaneity thresholds did not differ between participants who participated in mindfulness meditation and those who listened to music. There were, however, significant differences in thresholds related to the synchrony or asynchrony of target-bar onset with thresholds overall lower following asynchronous premask presentation, and with a reduction in the number of reports of simultaneous luminance change following asynchronous relative to synchronous bar onset, this being the bias effect discussed earlier. This pattern of effects has been reported in some previous studies (i.e., Elliott \& Shanagher, 2010; Giersch et al., 2009; Schmidt et al., 2011). This was not observed in the original experiment of Elliott et al. (2007), in which thresholds did not differ by premask, although the bias against reporting simultaneity was found, and was limited to luminance changes separated by up to $21 \mathrm{~ms}$. This led to differences in the slope of the psychometric function and the possibility that asynchronous bar onsets were coded by a slightly different system, to the synchronous bar onsets. 
(a)

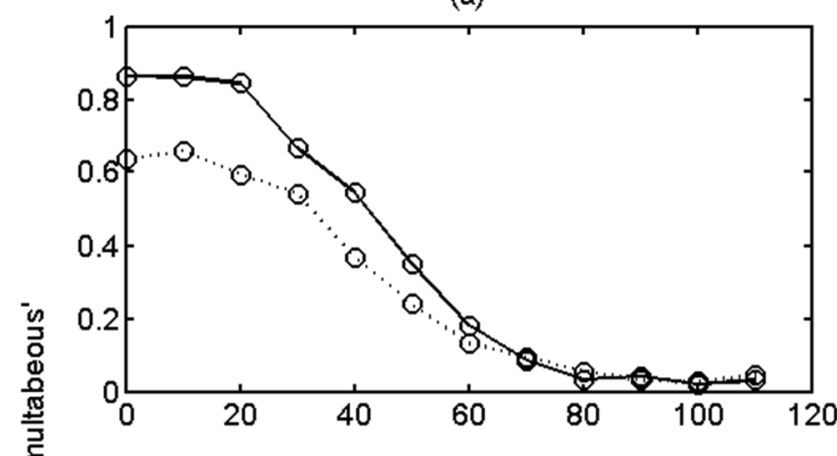

(c)

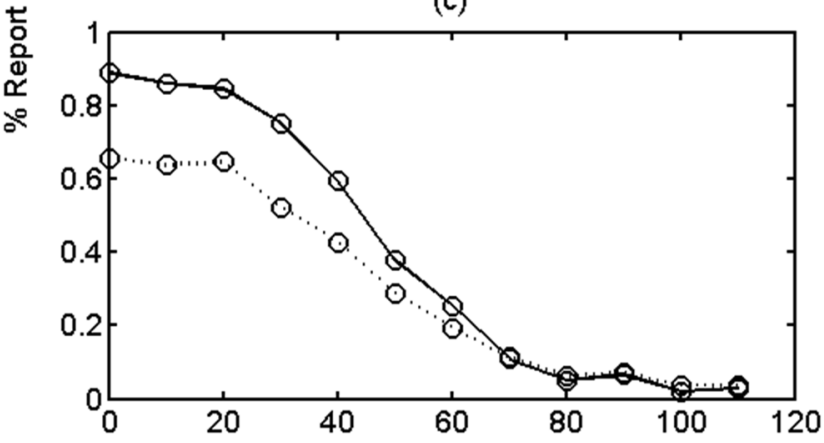

(b)

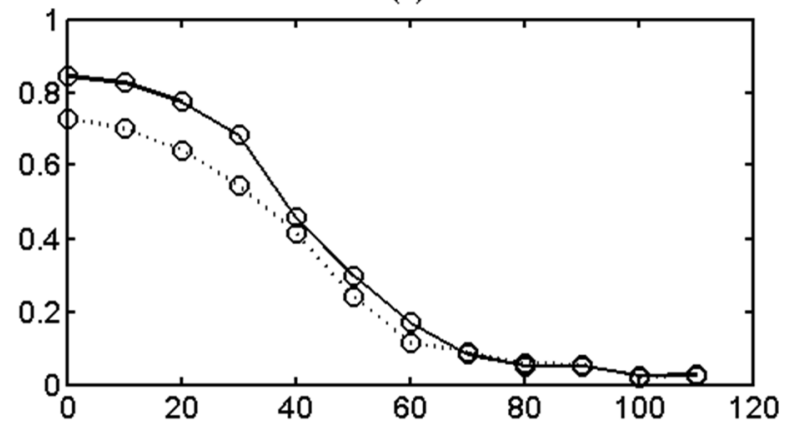

(d)

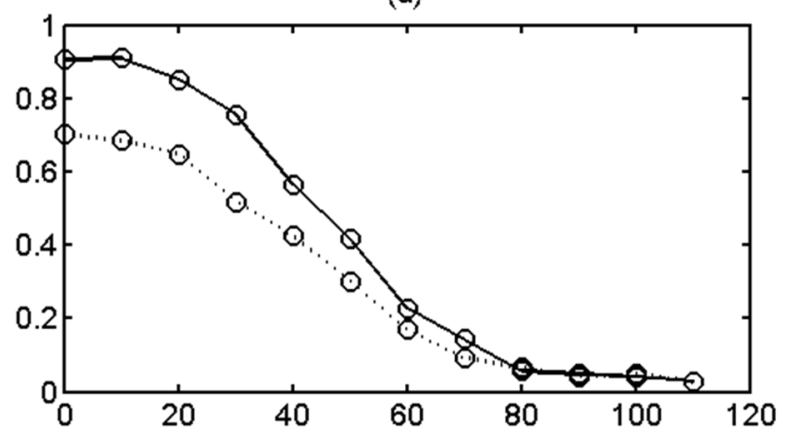

ISI (ms)

Fig. 3 Psychometric functions describing the grand-mean percentages of "simultaneous responses" by premask condition (continuous line synchronous, dashed line asynchronous) over ISI, for (a) mindfulness condition pre-intervention; (b) mindfulness condition post-intervention; (c) control condition pre-intervention and (d) control condition post-intervention. Although not associated with a significant increase in slope relative to the other conditions, in the

Differences in slope found in the experiment reported here would suggest the same, although this seems uninfluenced by mindfulness meditation.

Interestingly, the mindfulness meditation intervention did bring about a small but significant reduction in the bias resulting from asynchronous onsets, leading to a significantly greater number of reports "simultaneous" following asynchronous bar presentation following mindfulness intervention, relative to the other conditions. This in turn resulted in the thresholds between synchronous and asynchronous bar-onset conditions being statistically indistinguishable following the mindfulness intervention. In all other conditions, thresholds between these two conditions differed significantly: this included thresholds in the same participant data being significantly different prior to the mindfulness intervention, while non-significantly different after intervention (following asynchronous bar onsets). Generally speaking, it might be argued that the asynchronous premask actually improves report accuracy because a reduced tendency to respond "simultaneous" alongside the lowering the threshold mindfulness condition post-intervention, there is a reduction in bias against reporting "simultaneous" following presentation of an asynchronous premask and at short ISIs. This bias reduction is associated with a non-significant difference between simultaneity thresholds by premask condition, which is not observed in any of the other pre- or post-intervention conditions

is the same thing as approaching the veridical report simultaneous, which is only true for the $0 \mathrm{~ms}$ ISI condition. However, responses "simultaneous" were also reduced when bars changed luminance at the same time, which tends to suggest that the pattern of effects found here needs to be considered in the context of the broader response profile.

In this context and in the data presented by Elliott et al. (2007), it could be argued that the change in slope of the psychometric function following asynchronous bar onsets served to maintain the threshold at the same point, irrespective to the effects of asynchronous onset. Attention and perception probably function by virtue of the synchronization of relevant visual brain mechanisms (see Singer, 1999 for an outline of this view), which suggests itself as a candidate psychophysiological process for mediating the content structure and therefore the duration of psychological moment. von Uexküll (1957) noted that the psychological moment, which differs between species (e.g., see Brecher, 1932), differs as a function of its capacity for the processing of aspects for the environment that are relevant to the animal 
(see also Elliott, 2019). A small stimulus asynchrony might be sufficient to disturb this synchronization, and this might explain why there is a bias against reporting the two stimuli to brighten simultaneously at short bar onset asynchronies. So, what happens following mindfulness intervention?

Mindfulness is thought of in terms of an endogenous attentional focalization (Kabat-Zinn, 1994, 2003), which is consistent with the usual idea of attention as a cognitive brain mechanism which focalizes either thought or perception to allow for more detailed processing. In addition, and related more directly to the present paradigm, mindfulness has been found to improve the engagement of sustained attention to response (by a reduction in mind wandering) (Mrazek et al., 2012). By this conceptualization, attention usually deploys to resolve detail in stimulus events (or to solve mental problems in contexts) that have been subject to a basic level of processing according to the nature or identity of the stimulus. This is at least consistent with the idea of a relationship between the duration of the moment and attention because focal attention is usually understood to deploy on the basis of the prospective relevance of the to be processed stimulus. Attention, by this conceptualization, is also related to the efficiency of low-level synchronization; either attention deploys based upon successful processing at the lower level, or it is itself responsible for organizing successful processing. A relationship between the structure and efficiency of functional connectivity of intrinsic brain networks, mindfulness, and sustained attention has been shown by Mooneyham et al. (2017). In the present context, mindfulness, in as much as this "conditions" an attentional response, may very well influence the organization or normalization of the brain processes responsible for that response, limiting the impact of asynchronous bar presentation and thereby resulting in only limited changes to the threshold, and thus only a small variation on the psychological moment. Considering the data of psychotic patients (Giersch et al., 2009; Schmidt et al., 2011) for whom the psychological moment is very substantially altered, this account suggests that there would be a limited physiological capacity for change in the duration of moment in healthy participants, while more substantial changes might be expected to accompany a psychopathology (see also the reduction in thresholds using a similar paradigm, in young adults with autism as reported by Falter et al., 2012). Although it might be argued that this study is powered to detect larger effects, leaving any nonsignificant results inconclusive, the sample size used in the present study was similar to that used in previous studies. These studies employed the same paradigm and found simultaneity thresholds located at around $50 \mathrm{~ms}$, as well as variations in reports "simultaneous" at shorter ISIs as a function of the synchrony or asynchrony of the bar onsets (e.g., Elliott et al., 2007; Giersch, et al., 2009).
In summary, mindfulness meditation has a small but significant effect on the efficiency of the mechanisms responsible for low-level visual processing, although and in contrast to a previous finding there is no evidence that this effect concerns a change in the structure of the mechanisms responsible for processing stimulus events, because the slopes of the psychometric functions are statistically indistinguishable across conditions. Although not tested directly in this study, we propose this effect might be one of improving the efficiency of neural synchronization, which relates both to attentional focus, and the content structure of the psychological present. We suggest that mindfulness meditation can be used to assist in rehabilitative therapy for disorders or conditions that influence the duration of the perceptual moment, for example psychotic disorder, or autism (see Elliott \& Giersch, 2016; Falter et al., 2012). In both cases, the disorder might of itself be exacerbated by variations in the duration of the moment. Furthermore, the effect of brief mindfulness meditation on the duration of the moment, and its effect upon sustained attention (Mrazek et al., 2012) and other cognitive functions such as working memory capacity (Mrazek et al., 2013) and more particularly the possibility that mind wandering may be reduced to improve these cognitive functions requires further consideration. Future research is recommended to examine these ideas.

Author Contribution The study was conceived by Mark A. Elliott, Monika Zalewska, and Marc Wittmann and carried out by Monika Zalewska; the data were analyzed by Mark A. Elliott and Monika Zalewska; and the manuscript was written by Mark A. Elliott, Monika Zalewska, and Marc Wittmann.

Funding Open Access funding provided by the IReL Consortium.

Data availability The datasets generated during and/or analyzed during the current study are available from the corresponding author on reasonable request.

\section{Declarations}

Ethical approval The study was approved by the National University of Ireland, Galway Research Ethics Committee, and informed written consent was obtained from all participants. The study was performed in accordance with the ethical standards as laid down in the 1964 Declaration of Helsinki and its later amendments.

Informed consent Informed consent was obtained from all individual participants included in the study.

Conflict of interest The authors declare no competing interests.

Open Access This article is licensed under a Creative Commons Attribution 4.0 International License, which permits use, sharing, adaptation, distribution and reproduction in any medium or format, as long as you give appropriate credit to the original author(s) and the source, provide a link to the Creative Commons licence, and indicate if changes 
were made. The images or other third party material in this article are included in the article's Creative Commons licence, unless indicated otherwise in a credit line to the material. If material is not included in the article's Creative Commons licence and your intended use is not permitted by statutory regulation or exceeds the permitted use, you will need to obtain permission directly from the copyright holder. To view a copy of this licence, visit http://creativecommons.org/licenses/by/4.0/.

\section{References}

Alexander, C. N., Rainforth, M. V., \& Gelderloos, P. (1991). Transcendental meditation, self-actualization, and psychological health: A conceptual overview and statistical meta-analysis. Journal of Social Behavior \& Personality, 6, 189-248.

Barratt, E. S., Stanford, M. S., Dowdy, L., Liebman, M. J., \& Kent, T. A. (1999). Impulsive and premeditated aggression: A factor analysis of self-reported acts. Psychiatry Research, 86, 163-173.

Berkovich-Ohana, A., Glicksohn, J., \& Goldstein, A. (2011). Temporal cognition changes following mindfulness, but not transcendental meditation practice. Proceedings of Fechner Day, 27, 245-250.

Berkovich-Ohana, A., Dor-Ziderman, Y., Glicksohn, J., \& Goldstein, A. (2013). Alterations in the sense of time, space, and body in the mindfulness-trained brain: A neurophenomenologically-guided MEG study. Frontiers in Psychology, 4, 912. https://doi.org/10. 3389/fpsyg.2013.00912

Berkovich-Ohana, A., \& Wittmann, M. (2017). A typology of altered states according to the consciousness state space (CSS) model. A Special Reference to Subjective Time. Journal of Consciousness Studies, 24, 37-61.

Brecher, G. A. (1932). Die Entstehung und biologische Bedeutung der subjectktiven Zeiteinheit-des Momentes. Zeitschrift Für Vergleichende Physiologie, 18, 204-243.

Carter, O. L., Presti, D. E., Callistemon, C., Ungerer, Y., Liu, G. B., \& Pettigrew, J. D. (2005). Meditation alters perceptual rivalry in Tibetan Buddhist monks. Current Biology, 15, 412-413.

Droit-Volet, S., Fanget, M., \& Dambrun, M. (2015). Mindfulness meditation and relaxation training increases time sensitivity. Consciousness and Cognition, 31, 86-97. https://doi.org/10.1016/j. concog.2014.10.007

Droit-Volet, S., \& Heros, J. (2017). Time judgments as a function of mindfulness meditation, anxiety and mindfulness awareness. Mindfulness, 8, 266-275. https://doi.org/10.1007/ s12671-016-0597-6

Droit-Volet, S., \& Dambrun, M. (2019). Awareness of the passage of time and self-consciousness: What do meditators report? PsyCh Journal, 8, 51-65. https://doi.org/10.1002/pchj.270

Elliott, M. A. (2019). Time opined: A being in the moment. In V. Arstila, A. Bardon, S. Power, \& A. Vatakis (Eds.), The illusions of time: Philosophical and psychological essays on timing and time perception (pp. 261-274). Palgrave Macmillan.

Elliott, M. A., Shi, Z., \& Kelly, S. D. (2006). A moment to reflect upon perceptual synchrony. Journal of Cognitive Neuroscience, 18(10), 1880-1883.

Elliott, M. A., Shi, Z., \& Sürer, F. (2007). The effects of subthreshold synchrony on the perception of simultaneity. Psychological Research Psychologische Forschung, 71, 687-693. https://doi.org/ 10.1007/s00426-006-0057-3

Elliott, M. A., \& Giersch, A. (2016). What happens in a moment. Frontiers in Psychology, 6, 1905. https://doi.org/10.3389/fpsyg.2015. 01905
Elliott, M. A., \& Shanagher, L. (2010). Temporal event-structure coding in developmental dyslexia: Evidence from explicit and implicit temporal processing. Psihologija, 43(4), 373-387.

Falter, C. M., Elliott, M. A., \& Bailey, A. J. (2012). Enhanced visual temporal resolution in autism spectrum disorders. PLOS ONE, 7(3), e32774. https://doi.org/10.1371/journal.pone.0032774

Giersch, A., Lalanne, L., Corves, C., Seubert, J., Shi, Z., Foucher, J., \& Elliott, M. A. (2009). Extended visual simultaneity thresholds in patients with schizophrenia. Schizophrenia Bulletin, 35, 816-825. https://doi.org/10.1093/schbul/sbn016

Giersch, A., Lalanne, L., van Assche, M., \& Elliott, M. A. (2013). On disturbed time continuity in schizophrenia: An elementary impairment in visual perception? Frontiers in Psychology, 4, 281. https:// doi.org/10.3389/fpsyg.2013.00281

Giersch, A., Poncelet, P., Capa, R. L., Martin, B., Duval, C. Z., Curzietti, M., \& Hoonacker, van Assche, M., \& Lalanne, L. . (2015). Disruption of information processing in schizophrenia: The time perspective. Schizophrenia Research: Cognition, 2, 78-83. https:// doi.org/10.1016/j.scog.2015.04.002

Grondin, S. (2001). From physical time to the first and second moments of psychological time. Psychological Bulletin, 127(1), 22.

Hölzel, B. K., Lazar, S. W., Gard, T., Schuman-Olivier, Z., Vago, D. R., \& Ott, U. (2011). How does mindfulness meditation work? Proposing mechanisms of action from a conceptual and neural perspective. Perspectives on Psychological Science, 6, 537-559. https://doi.org/10.1177/1745691611419671

Husserl, E. (1928). Vorlesungen zur Phänomenologie des inneren Zeitbewußtseins. Max Niemeyer Verlag.

Kabat-Zinn, J. (1994). Wherever you go, there you are: Mindfulness meditation in everyday life. New York, NY: Hyperion.

Kabat-Zinn, J. (2003). Mindfulness-based interventions in context: Past, present and future. Clinical Psychology Science and Practice, 10, 144-156. https://doi.org/10.1093/clipsy/bpg016

Kramer, R. S. S., Weger, U. W., \& Sharma, D. (2013). The effect of mindfulness meditation on time perception. Consciousness and Cognition, 22, 846-852. https://doi.org/10.1016/j.concog.2013. 05.008

Matko, K., Ott, U., \& Sedlmeier, P. (2021). What do meditators do when they meditate? Proposing a novel basis for future meditation research. Mindfulness, 12, 1791-1811. https://doi.org/10. 1007/s12671-021-01641-5

Mooneyham, B. W., Mrazek, M. D., Mrazek, A. J., Mrazek, K. L., Phillips, D. T., \& Schooler, J. W. (2017). States of mind: Characterizing the neural bases of focus and mind-wandering through dynamic functional connectivity. Journal of Cognitive Neuroscience, 29(3), 495-506.

Mrazek, M. D., Smallwood, J., \& Schooler, J. W. (2012). Mindfulness and mind-wandering: Finding convergence through opposing constructs. Emotion, 12, 442. https://doi.org/10.1037/a0026678

Mrazek, M. D., Franklin, M. S., Phillips, D. T., Baird, B., \& Schooler, J. W. (2013). Mindfulness training improves working memory capacity and GRE performance while reducing mind wandering. Psychological Science, 24(5), 776-781.

Sauer, S., Lemke, J., Wittmann, M., Kohls, N., Mochty, U., \& Walach, H. (2012). How long is now for mindfulness meditators? Personality and Individual Differences, 52, 750-754. https://doi.org/10. 1016/j.paid.2011.12.026

Sauer, S., Ziegler, M., Danay, E., Ives, J., \& Kohls, N. (2013). Specific objectivity of mindfulness - A Rasch analysis of the Freiburg Mindfulness Inventory. Mindfulness, 4, 45-54.

Schäfer, T., Fachner, J., \& Smukalla, M. (2013). Changes in the representation of space and time while listening to music. Frontiers in Psychology, 4, 508.

Scheufele, P. M. (2000). Effects of progressive relaxation and classical music on measurements of attention, relaxation, and stress responses. Journal of Behavioral Medicine, 23, 207-228. 
Schmidt, H., McFarland, J., Ahmed, M., McDonald, C., \& Elliott, M. A. (2011). Low-level temporal coding impairments in psychosis: Preliminary findings and recommendations for further studies. Journal of Abnormal Psychology, 120, 476-482. https://doi.org/ 10.1037/a0023387

Sedlmeier, P., Winkler, I., \& Lukina, A. (2020). How long did the time spent in meditation feel? "Attention. Attention. Attention.". Psychology of Consciousness: Theory, Research, and Practice, https://doi.org/10.1037/cns0000254

Singer, W. (1999). Neuronal synchrony: A versatile code for the definition of relations? Neuron, 24(1), 49-65.

Thönes, S., \& Wittman, M. (2016). Time perception in yogic mindfulness meditation-Effects on retrospective duration judgments and time passage. Psychology of Consciousness: Theory, Research, and Practice, 3, 316-325. https://doi.org/10.1037/cns0000088

Treutwein, B. (1995). Adaptive psychophysical procedures. Vision Research, 35, 2503-2522.

Vaitl, D., Birbaumer, N., Gruzelier, J., Jamieson, G. A., Kotchoubey, B., Kübler, A., Lehmann, D., Miltner, W. H. R., Ott, U., \& Pütz, P. (2005). Psychobiology of altered states of consciousness. Psychological Bulletin, 131, 98-127. https://doi.org/10.1037/00332909.131.1.98

von Uexküll, J.B. (1957) A stroll through the worlds of animals and men: A picture book of invisible worlds. In Claire H. Schiller (Ed.) Instinctive behavior: The development of a modern concept: New York: International Universities Press. pp. 5-80.

Walach, H., Buchheld, N., Buttenmüller, V., Kleinknecht, N., \& Schmidt, S. (2006). Measuring mindfulness-The Freiburg mindfulness inventory (FMI). Personality and Individual Differences, 40, 1543-1555. https://doi.org/10.1016/j.paid.2005.11.025
Weiner, L., Wittmann, M., Bertschy, G., \& Giersch, A. (2016). Dispositional mindfulness and subjective time in healthy individuals. Frontiers in Psychology, 7, 786. https://doi.org/10.3389/fpsyg. 2016.00786

Wittmann, M., \& Paulus, M. P. (2008). Decision making, impulsivity and time perception. Trends in Cognitive Sciences, 12, 7-12. https://doi.org/10.1016/j.tics.2007.10.004

Wittmann, M., \& Schmidt, S. (2014). Mindfulness meditation and the experience of time. In S. Schmidt, \& H. Walach (Eds.), Meditation - Neuroscientific approaches and philosophical implications. Studies in neuroscience, consciousness and spirituality, 2. Cham: Springer-Verlag.

Wittmann, M., Peter, J., Gutina, O., Otten, S., Kohls, N., \& Meissner, K. (2014). Individual differences in self-attributed mindfulness levels are related to the experience of time and cognitive selfcontrol. Personality and Individual Differences, 64, 41-45. https:// doi.org/10.1016/j.paid.2014.02.011

Wittmann, M. (2015). Modulations of the experience of self and time. Consciousness and Cognition, 38, 172-181. https://doi.org/10. 1016/j.concog.2015.06.008

Wittmann, M., Otten, S., Schötz, E., Sarikaya, A., Lehnen, H., Jo, H. G., Kohls, N., Schmidt, S., \& Meissner, K. (2015). Subjective expansion of extended timespans in experienced meditators. Frontiers in Psychology, 5, 1581. https://doi.org/10.3389/fpsyg. 2014.01586

Publisher's note Springer Nature remains neutral with regard to jurisdictional claims in published maps and institutional affiliations. 